ARTICLE

DOI: $10.1038 /$ s41467-018-06103-6

\title{
Gut fungal dysbiosis correlates with reduced efficacy of fecal microbiota transplantation in Clostridium difficile infection
}

Tao Zuo ${ }^{1,2}$, Sunny H. Wong1,2,3, Chun Pan Cheung ${ }^{1}$, Kelvin Lam¹, Rashid Lui ${ }^{1}{ }^{1}$, Kitty Cheung ${ }^{1}$ Fen Zhang ${ }^{4}$, Whitney Tang1, Jessica Y.L. Ching ${ }^{1}$, Justin C.Y. Wu ${ }^{1,2}$, Paul K.S. Chan ${ }^{3,5}$, Joseph J.Y. Sung 1,2, Jun Yu (1) 1,2,3, Francis K.L. Chan ${ }^{1,2,3} \&$ Siew C. $\mathrm{Ng}^{1,2,3}$

Fecal microbiota transplantation (FMT) is effective in treating recurrent Clostridium difficile infection (CDI). Bacterial colonization in recipients after FMT has been studied, but little is known about the role of the gut fungal community, or mycobiota. Here, we show evidence of gut fungal dysbiosis in CDI, and that donor-derived fungal colonization in recipients is associated with FMT response. CDI is accompanied by over-representation of Candida albicans and decreased fungal diversity, richness, and evenness. Cure after FMT is associated with increased colonization of donor-derived fungal taxa in recipients. Recipients of successful FMT ("responders") display, after FMT, a high relative abundance of Saccharomyces and Aspergillus, whereas "nonresponders" and individuals treated with antibiotics display a dominant presence of Candida. High abundance of C. albicans in donor stool also correlates with reduced FMT efficacy. Furthermore, $C$. albicans reduces FMT efficacy in a mouse model of $\mathrm{CDI}$, while antifungal treatment reestablishes its efficacy, supporting a potential causal relationship between gut fungal dysbiosis and FMT outcome.

\footnotetext{
${ }^{1}$ Department of Medicine and Therapeutics, The Chinese University of Hong Kong, Hong Kong, China. ${ }^{2}$ Institute of Digestive Disease, State Key Laboratory of Digestive Diseases, LKS Institute of Health Science, The Chinese University of Hong Kong, Hong Kong, China. ${ }^{3}$ Center for Gut Microbiota Research, The Chinese University of Hong Kong, Hong Kong, China. ${ }^{4}$ Shenzhen Research Institute, The Chinese University of Hong Kong, Shenzhen, China. ${ }^{5}$ Department of Microbiology, The Chinese University of Hong Kong, Hong Kong, China. Correspondence and requests for materials should be addressed to

S.C.N. (email: siewchienng@cuhk.edu.hk)
} 
T he past decade has witnessed an increasing use of fecal microbiota transplantation (FMT) as a promising treatment option for several diseases ${ }^{1-3}$, yet success rates are variable with a cure rate of $85-90 \%$ in recurrent Clostridium difficile infection $(\mathrm{CDI})^{3-5}$, and a response rate of $30-50 \%$ in inflammatory bowel disease (IBD) ${ }^{6-8}$. Such variations may be related to disease traits, recipient factors or donor characteristics. The mechanisms underlying a successful FMT and its relationship with gut microbial profiles in donor-recipient pairs remain elusive. To date, the efficacy of FMT has been mostly ascribed to the restoration of a normal bacterial microbiota composition and function, and a sustained coexistence of donor and recipient bacterial strains $s^{9-12}$. Recently, bacteriophages have been shown to be altered in CDI after FMT and these changes were associated with treatment outcome ${ }^{13-15}$. The human gastrointestinal tract is also colonized by a large population of fungi, collectively referred to as the mycobiota, which play an important role in human health ${ }^{16,17}$. Our gut mycobiota contributes to normal human physiology and in some cases can recapitulate the benefit of intestinal bacteria via regulating host immunity and maintaining intestinal homeostasis ${ }^{16-18}$. Whether donor-derived mycobiota can colonize a recipient host, the fate of donor and recipient mycobiota after FMT and their relationship with treatment outcomes are unknown.

Here, we study the gut mycobiota of FMT-treated subjects with CDI to explore the effects of FMT on the gut mycobiome in association with treatment outcome. We show enteric mycobiota dysbiosis in CDI subjects, and that cure after FMT is more frequently observed when donor-derived fungal taxa predominate in the recipient mycobiota. The gut mycobiome of FMT nonresponders and antibiotic-treated CDI subjects displays a dominant presence of Candida and Candida albicans after treatment. Hence, C. albicans abundance is associated with unfavorable FMT outcome in humans. Furthermore, we show that C. albicans compromises FMT efficacy in a mouse model of CDI.

\section{Results}

Gut fungal dysbiosis in CDI. We compared the fecal mycobiomes of 31 CDI subjects and 24 healthy controls. The total fecal fungal load was higher in CDI than in controls [Mann-Whitney test, $p=0.0004, \log _{10}$ transformed effect size 1.32 (95\% CI: $0.62-1.97$ ), Fig. 1a]. However, there was a decrease in fungal diversity, evenness, and richness in CDI compared with controls (Mann-Whitney test, $p=0.0120,0.0309$, and 0.0043 , respectively, Fig. 1b). The fungal communities of CDI subjects were separated from those of healthy controls at the operational taxonomic unit (OTU) level (based on Bray-Curtis dissimilarities, adonis test $p=0.003$, Fig. 1c). At the phylum level, Ascomycota was expanded in CDI compared with controls (Mann-Whitney test, $p=0.0083$, Fig. 1d). At the species level, 17 fungal species were found to be differentially present between $\mathrm{CDI}$ and controls (LefSe analysis with false discovery rate (FDR) adjusted $q<0.05$, Fig. 1e and Supplementary Data 1). Among these species, only C. albicans was significantly enriched in CDI (Fig. 1e, f, Mann-Whitney test, $p=0.0080$ ), whereas 16 other species were enriched in controls. We then used quantitative PCR to determine the absolute abundance of C. albicans in feces and confirmed the over-presentation of $C$. albicans in CDI [Mann-Whitney test, $p<0.0001, \log _{10}$ transformed effect size 1.53 (95\% CI: 0.90-2.11), Supplementary Figure 1a].

Antibiotic use has been shown to be a major contributor to the development of CDI by decreasing bacterial colonization resistance ${ }^{19,20}$. Antibiotic use has also been reported to promote the growth of C. albicans ${ }^{21,22}$. We further assessed the effect of antibiotics on C. albicans levels in CDI. We collected stool samples from consecutive new CDI patients, including 12 CDI patients with antibiotics exposure, 12 CDI patients with no antibiotics exposure at inclusion, and 17 healthy controls. We found significantly higher levels of fecal C. albicans in CDI subjects exposed to antibiotics at inclusion, compared with controls (Supplementary Figure 1b, Mann-Whitney test, $p=$ 0.0131). C. albicans levels were also higher in CDI subjects not exposed to antibiotics at inclusion when compared with controls (Supplementary Figure 1b, Mann-Whitney test, $p=0.0469$ ). These data suggest that antibiotics may not be the only contributor to increased levels of $C$. albicans and that $C$. difficile infection per se is also associated with C. albicans bloom.

In line with the observation at the species level, more taxa were enriched in controls than in CDI, as determined by LefSe analysis (9 versus 1 at the order level, 15 versus 2 at the family level, and 16 versus 1 at the genus level, Supplementary Data 2). Altogether, these data indicate dysbiosis of the enteric mycobiota in patients with CDI. MaAsLin analysis identified no significant correlations between discriminative fungal taxa and subject metadata (age, gender, household, CDI severity, and underlying IBD).

FMT alters the gut mycobiota in relation to treatment response. We then explored whether FMT leads to colonization of donor-derived fungi and its association with treatment efficacy. Changes in the gut mycobiomes of recipients after FMT were monitored at multiple time-points in 16 CDI subjects, using preFMT samples of each donor-recipient pair as baseline for FMT (Supplementary Figure 2). Among 16 CDI subjects treated with FMT, 9 remained symptom-free with a negative stool $C$. difficile toxin at the last follow-up (termed "responders", FMT1-FMT9), while seven developed recurrence of CDI (termed "nonresponders", FMT10-FMT16) (Supplementary Data 3). We next investigated whether donor-derived fungi and bacteria in recipients were associated with FMT outcomes. Subjects who responded to FMT demonstrated a larger proportion of fungal and bacterial OTUs that were transferred and predominated in the fecal microbiota of recipients after FMT, compared to those who did not respond (Mann-Whitney test, $p=0.0068$ and 0.0164 , respectively for comparisons of donor-derived fungal and bacterial OTU ratios in recipients, Supplementary Figure 3a, b).

During follow-up, FMT responders showed an increase in fungal richness and diversity after FMT (Wilcoxon matched-pairs singed rank test, $p=0.0273$ and $p=0.0474$, respectively, Fig. 2a, b). There were profound differences in the gut mycobiota configurations across different donor-recipient pairs following FMT. The relative abundance of the genus Candida was higher in FMT nonresponders compared with responders across serial post-FMT fecal samples (Fig. 3a, b). Discriminative analysis identified disparately presented fungal taxa between post-FMT samples of FMT responders and nonresponders, at the genus and species level (Fig. 3b). Post-FMT, the genera Saccharomyces, Aspergillus, and Penicillum were relatively more abundant in FMT responders than in nonresponders. At the species level, $C$. albicans was the most prominent species enriched in nonresponders after FMT.

We also investigated alterations in the bacterial microbiota both at baseline, between CDI and controls, and after FMT in CDI recipients. Bacterial diversity, evenness and richness at baseline were lower in CDI subjects than controls (Mann-Whitney test, all $p<0.01$, Supplementary Figure $4 a$ ). After FMT there was a significant increase in bacterial richness (Wilcoxon matched-pairs singed rank test, $p=0.019$ ) and a marginally significant increase in bacterial diversity (Wilcoxon matched-pairs singed rank test, $p=0.098$ ) in FMT responders (Supplementary Figure $4 \mathrm{~b}, \mathrm{c}$ ). We explored the composition of 


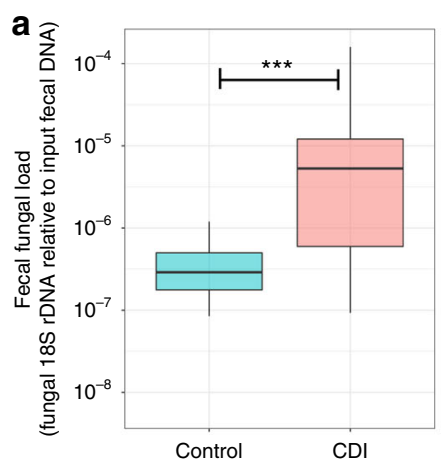

C b

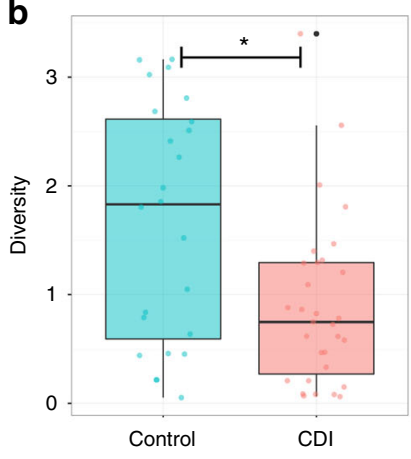

d
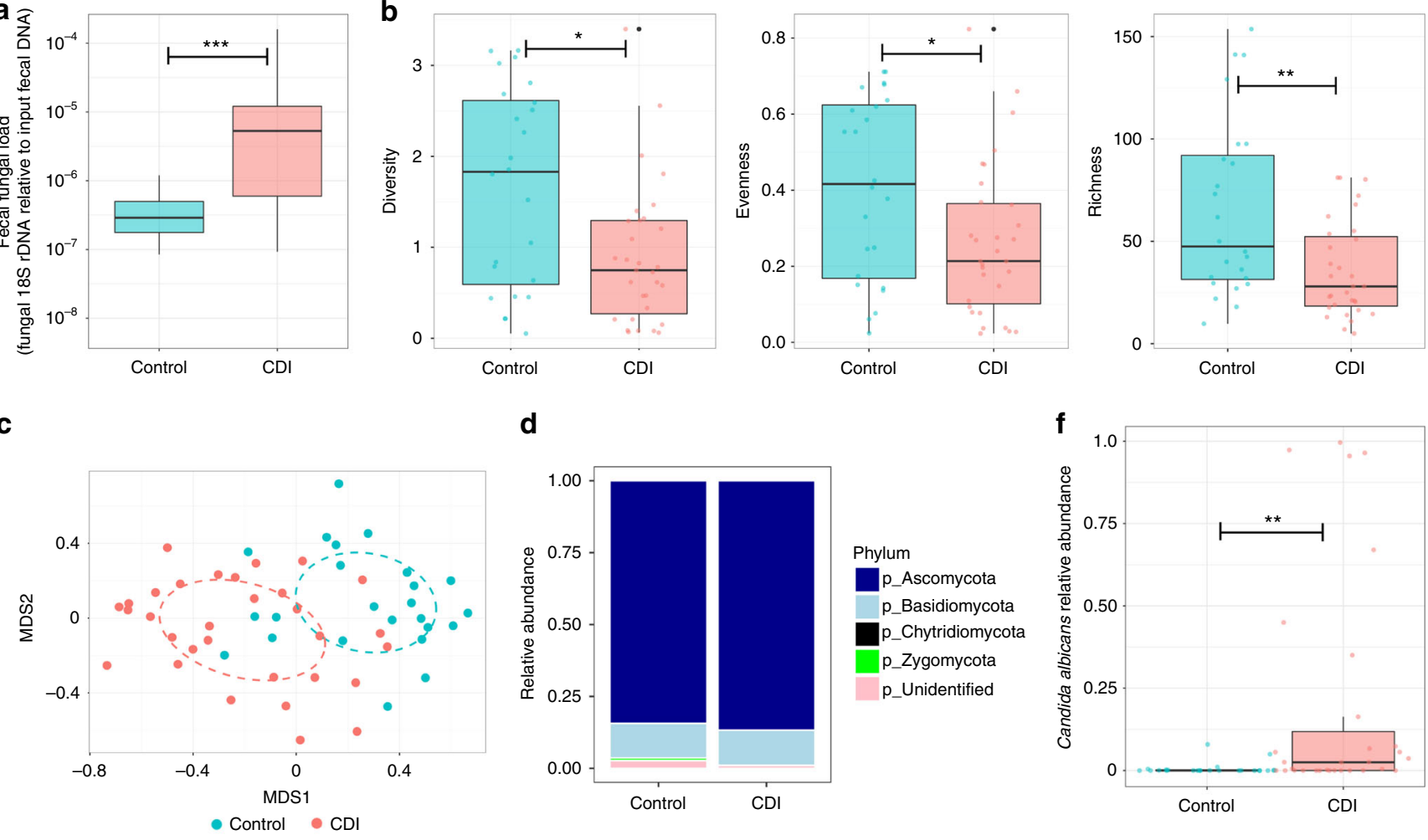

f

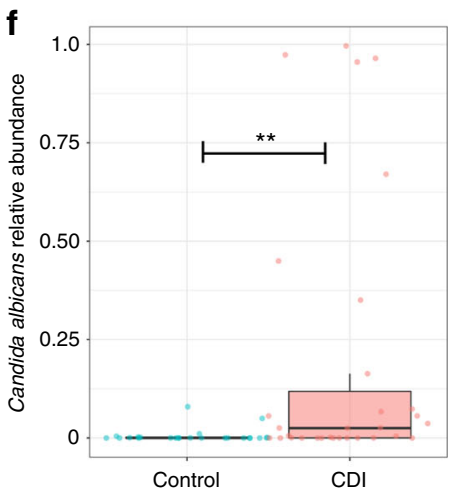

e

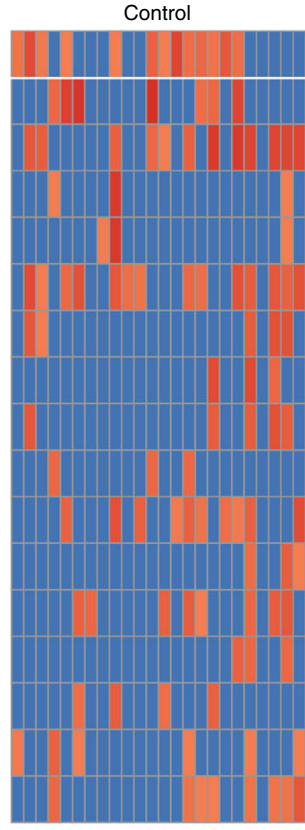

CDI
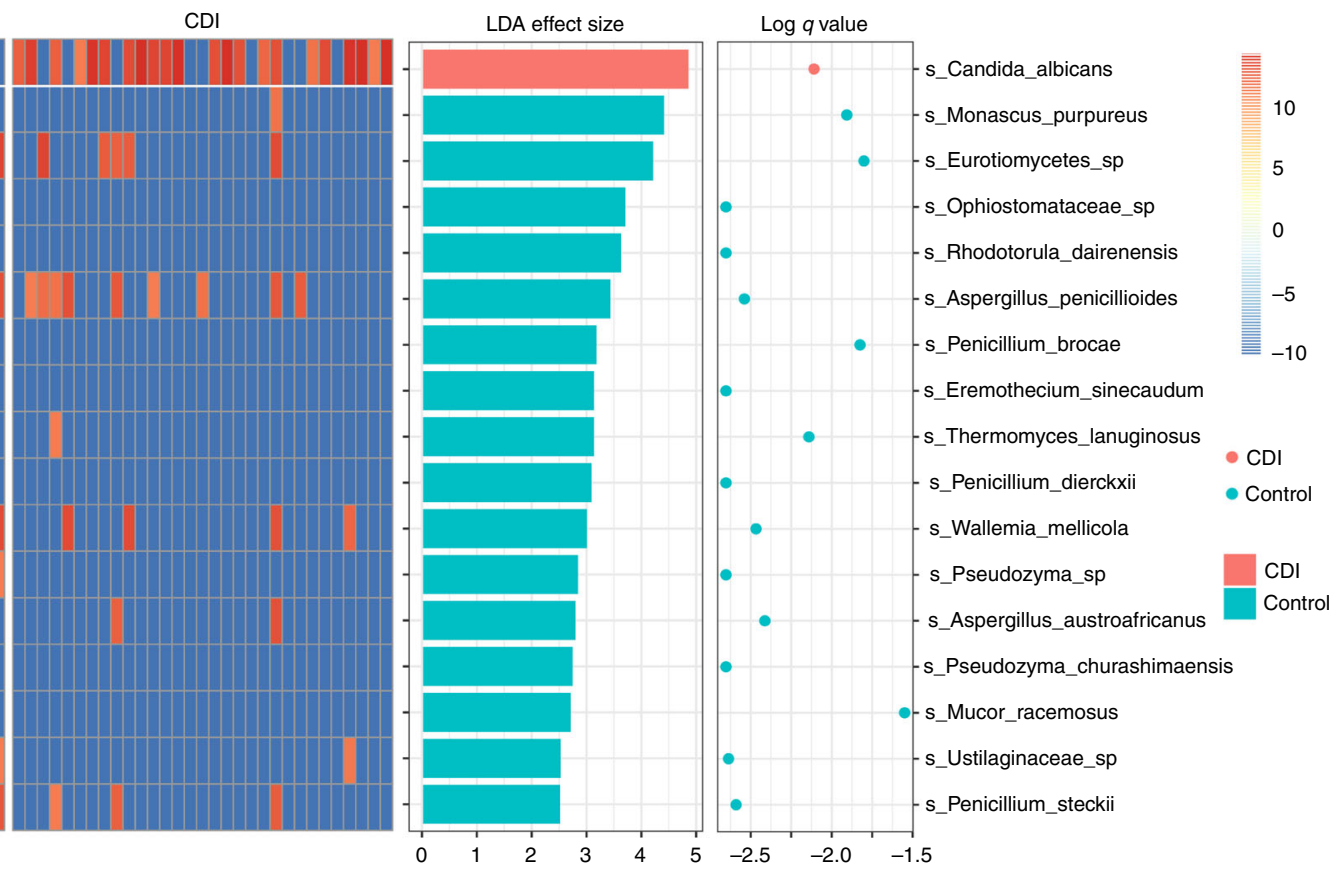

Fig. 1 Fungal alterations in CDI. a Comparison of the total fungal load in the feces of controls and CDI subjects. Statistical significance was determined by Mann-Whitney test, ${ }^{\star \star \star} P<0.001$. b Comparison of the fecal mycobiota based on Shannon diversity, evenness, Chao1 richness in controls and CDI subjects. The dots indicate individual values of the studied subjects. Statistical significance was determined by Mann-Whitney test, ${ }^{\star} P<0.05,{ }^{\star \star} P<0.01$. c Fungal community structure difference between controls and CDI by NMDS (nonmetric multidimensional scaling) plot based upon Bray-Curtis dissimilarities. $\mathbf{d}$ Comparison of the fecal mycobiota composition between controls and CDI subjects at the phylum level. e Differentially enriched fungal species between controls and CDI. Statistical significance was determined by LefSe analysis with FDR correction (only those species with $q$ values $<0.05$ and LDA effect size $>2$ are shown). Heatmap of the presence of these differential fungal species is shown in relative abundance intensity. LDA effect size, $\log _{10}$ transformed $q$ value (FDR-adjusted $p$ value) and species annotation are shown. Green bars and dots indicate species enriched in controls, while red bars and dots indicate species enriched in CDI. f Comparison of the relative abundance of fecal C. albicans in controls and CDI subjects. Statistical significance was determined by Mann-Whitney test, ${ }^{\star \star} P<0.01$. For box plots, the boxes extend from the first to third quartile (25th to 75 th percentile), with the median depicted by a vertical line 

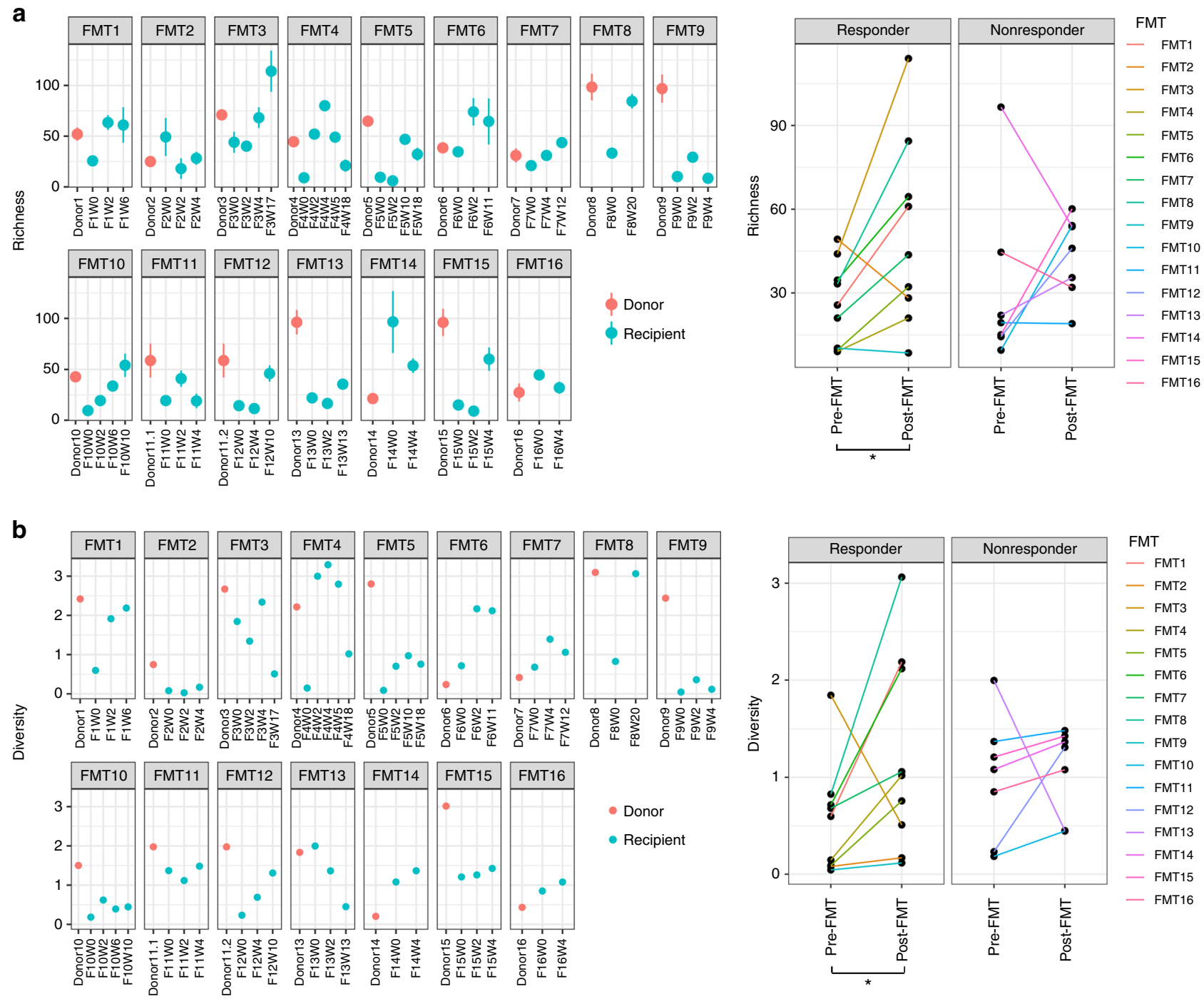

Fig. 2 Post-FMT alterations in the enteric mycobiota richness and diversity of CDI recipients in association with FMT response. Fecal fungal richness (a) and diversity (b) alterations in FMT recipients over the course of longitudinal follow-up and in their corresponding donors at baseline. Comparison of the fungal richness and diversity of pre-FMT samples and post-FMT samples collected at the last follow-up are shown in FMT responders and FMT nonresponders, respectively. Statistical significance was determined by paired Wilcoxon signed rank test, " $p<0.05$. "F" indicates FMT-treated subject. "W" indicates weeks post-treatment

the bacterial microbiota after FMT in relation to FMT outcomes, at various taxonomic levels (Supplementary Figure 5). The Actinobacteria and Bacteroidetes phyla, the Lachnospiraceae, Clostridiaceae, and Ruminococcaceae families, and the Clostridium, Blautia, and Faecalibacterium genera were enriched in FMT responders in comparison with nonresponders after FMT. However, bacteria from the phylum Proteobacteria were more abundant in FMT nonresponders relative to FMT responders. FMT responders displayed bacterial abundances resembling that of the donor, whereas FMT nonresponders showed inadequate relative abundances of donor-enriched bacteria at the last followup after FMT (Supplementary Figure 5c). In recipients FMT12 and FMT16, bacterial configurations at the last follow-up after FMT were similar to that of healthy individuals, but their gut mycobiota configurations differed significantly from that of healthy individuals (Fig. 3a). Taken together, these data suggest that the final proportion of donor-derived fungal and bacterial taxa and alterations of the fecal fungal composition in the recipient post-FMT are associated with FMT outcome.
C. albicans is associated with poor FMT outcome. The relative abundance of $C$. albicans within the fecal fungal community was decreased after FMT (Wilcoxon matched-pairs singed rank test, $p=0.0458$, Fig. 4a). Recipients with an initial high relative abundance of $C$. albicans before FMT and who continued to have a high relative abundance of $C$. albicans $>10 \%$ after FMT experienced disease recurrence after FMT (Fig. 4a). In accordance, the absolute abundance of C. albicans (in fecal input DNA) was markedly decreased after FMT in FMT responder group (Wilcoxon matched-pairs singed rank test, $p=0.0391$, Fig. $4 \mathrm{~b}$ ). Interestingly, FMT nonresponders exhibited higher post-FMT fecal C. albicans levels in absolute abundance than FMT responders [Mann-Whitney test, $p=0.0018, \log _{10}$ transformed effect size 3.05 (95\% CI: 1.48-4.29), Fig. 4b]. Discriminative analysis via LefSe on the fecal mycobiomes between donors corresponding to FMT responders and nonresponders discerned C. albicans as the only significantly differential species enriched in FMT nonresponders' donor stool (least discriminant analysis (LDA) effect size 2.25, FDR-adjusted $q=0.029$ ). Accordingly, 
a
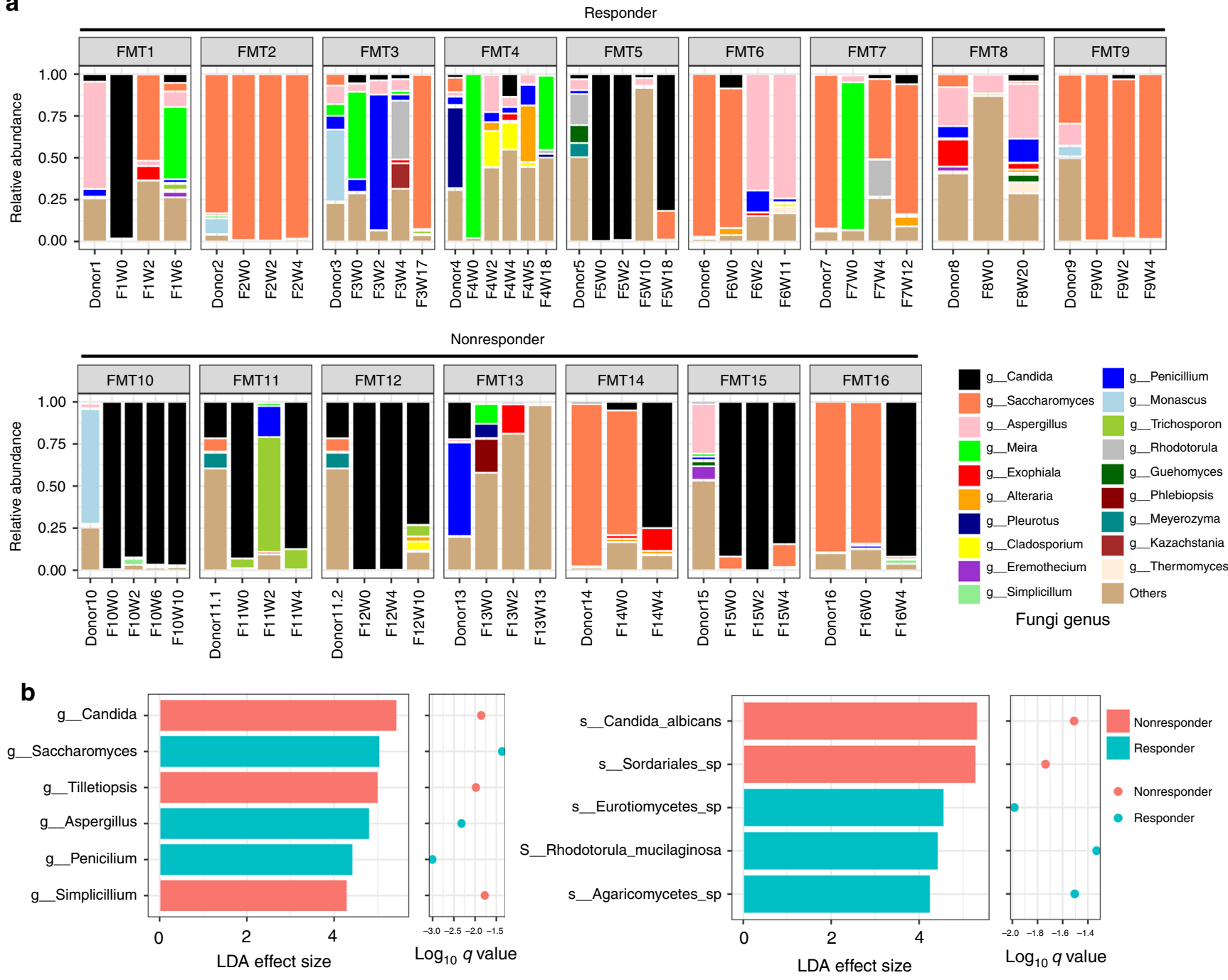

Fig. 3 Post-FMT alterations in the enteric mycobiota composition of CDI recipients in association with FMT response. a Alterations in the fecal fungal composition at the genus level in CDI recipients after FMT at different time-points up to the last follow-up. $\mathbf{b}$ Differentially enriched fungal taxa across all post-FMT fecal samples of FMT responders versus nonresponders at the genus and species levels. Statistical significance level was determined by LefSe analysis with FDR correction (only those taxa with $q$ values $<0.05$ and LDA effect size $>2$ are shown) and adjustment for subject number. Green bars and dots indicate taxa enriched in FMT responders, while red bar and dot indicate taxa enriched in FMT nonresponders

LefSe analysis on the fecal bacteriomes of donors at the genus level identified Escherichia and Proteus as the differentially enriched genera in FMT responders' donor stool and in FMT nonresponders' donor stool respectively (LDA effect size 2.58 and 2.35 , FDR-adjusted $q=0.017$ and 0.006 , respectively). These data demonstrated that the abundance of $C$. albicans both in postFMT recipient feces and in donor feces correlate with FMT outcome.

We subsequently screened and selected two new donors with undetectable fecal C. albicans, as determined by $\mathrm{qPCR}$, and transplanted their fecal preparations into five new CDI recipients (Supplementary Figure 6a). After FMT, four CDI recipients showed low fecal C. albicans levels (Supplementary Figure 6b). One recipient who continued to show high level of $C$. albicans after FMT (Case 1) had early recurrence of CDI (Supplementary Figure 6).

Antibiotic treatment leads to gut mycobiota alterations distinct from FMT treatment. We also assessed the effect of antibiotics on the gut mycobiota across longitudinal timepoints in eight CDI subjects treated with vancomycin (STD treatment, Supplementary Figure 2, Supplementary Data 3). Five of the eight subjects remained symptom-free with negative stool C. difficile toxins at last follow-up (termed "responders", STD1-STD5), while three developed recurrence of CDI (termed "nonresponders", STD6-STD8). Unlike FMT, vancomycin induced inconsistent alterations in the fungal richness and diversity during longitudinal follow-up (Supplementary Figure $7 \mathrm{a}, \mathrm{b})$. FMT and vancomycin led to an increase in the gut fungal diversity in $81.3 \%$ (13 out of 16 ) and $37.5 \%$ ( 3 out of 8 ) of CDI subjects respectively (chi-square test $p=0.032$, Supplementary Figure 7c), and an increase in the gut fungal richness in $68.8 \%$ (11 out of 16 ) and $37.5 \%$ (3 out of 8 ) of CDI subjects respectively (Supplementary Figure 7c). FMT responders showed a higher fold-change post-FMT in mycobiota richness when compared to STD responders (Student's $t$ test, $p$ $=0.0231$ ), while differences in mycobiota diversity between FMT responders and STD responders were not statistically significant (Supplementary Figure $7 d$, e). There was no 
a

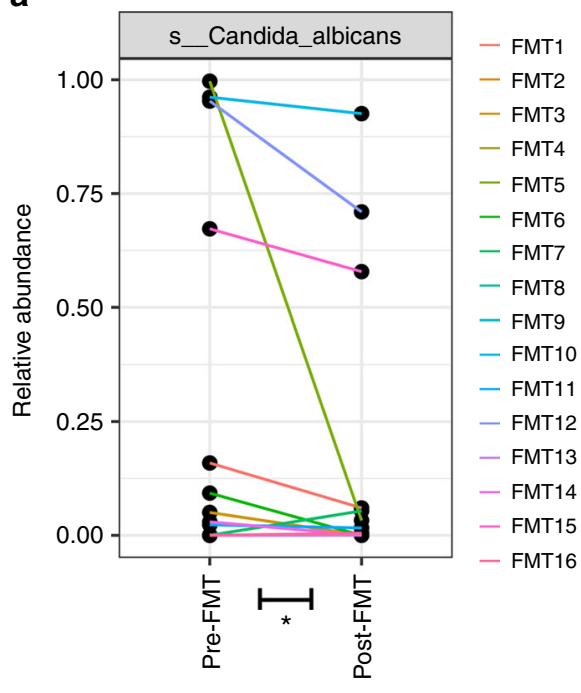

b

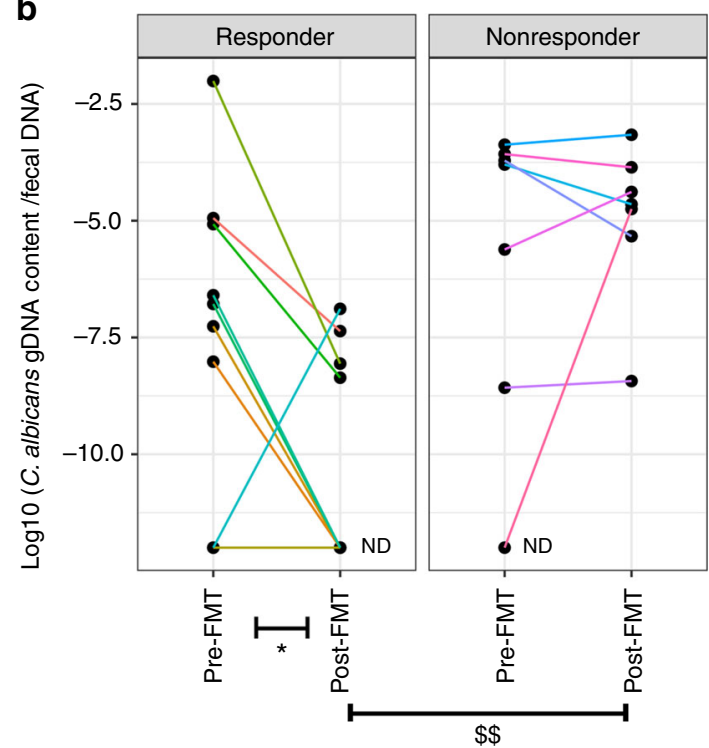

Fig. 4 The presence of $C$. albicans is linked to FMT outcomes in CDI. a Alterations in the relative abundance of fecal C. albicans before and after FMT at the last follow-up in FMT recipients, assessed by ITS sequencing. The relative abundance of fecal $C$. albicans was calculated as the proportion of sequences taxonomically binned as $C$. albicans relative to the sequences classified as fungi. Statistical significance was determined by paired Wilcoxon signed rank test, ${ }^{\star} p<0.05$. b The absolute fecal C. albicans levels before and after FMT at the last follow-up in FMT recipients, assessed by quantitative PCR. For those FMT nonresponders, the last follow-up time-point was a time prior to CDI recurrence after FMT. Comparison of the fecal $C$. albicans levels between preFMT samples and post-FMT samples was performed by paired Wilcoxon signed rank test, ${ }^{\star} p<0.05$. Comparison of the fecal $C$. albicans levels between the post-FMT samples of FMT responders and FMT nonresponders was performed by Mann-Whitney test, $\$ \$ p<0.01$. ND denotes no detectable C. albicans in the feces

significant difference in the bacterial richness or diversity between STD responders and nonresponders after vancomycin treatment, although vancomycin treatment resulted in a significant increase in bacterial diversity in STD responders (Supplementary Figure 8, Wilcoxon matched-pairs singed rank test, $p=0.0198$ ). Collectively, these data suggest that FMT may induce alterations in the gut mycobiota more effectively than antibiotics.

We further performed taxonomical analysis to study the effect of antibiotics on the fungal community and to determine differences between FMT and antibiotics treatment in modulating the gut mycobiota. After vancomycin treatment, fungal compositions exhibited similar configurations across nearly all recipients during follow-up with a marked increase of the genus Candida (Supplementary Figure 9a). Mice also demonstrated augmented fecal Candida levels post vancomycin treatment (Supplementary Figure 9b, Wilcoxon matched-pairs singed rank test, $p=0.0156$ ). There was no significant difference in the relative or absolute abundance of C. albicans between STD responders and nonresponders (Supplementary Figure 9c, d). Subject STD7, who showed a high post-STD relative abundance of C. albicans ( $>10 \%)$ and high absolute abundance in feces, developed CDI recurrence after vancomycin treatment, further supporting a correlation between C. albicans abundance and CDI outcome.

To define differentially enriched fungal taxa with respect to FMT and vancomycin treatment, we implemented LefSe analysis across all the follow-up samples of subjects corresponding to these two regimes. FMT treatment enriched the genera Saccharomyces and Cryptococcus in post-FMT samples, whereas vancomycin disparately enriched a panel of fungal genera after treatment, which included Candida, Talaromyces, Erythrobasidium, Periconia, Stemphylium, and Ganoderma (Supplementary Figure 10). At the family level, FMT was associated with enrichment of Saccharomycetacea and Herpotrichiellaceae (Supplementary Figure 10).

To characterize the ecological network of the gut mycobiota and bacterial microbiota, we evaluated the correlation of the $\alpha$ diversity (diversity, evenness, and richness) of the fungal community with that of the bacterial community. Among the post-treatment samples of FMT responders, significant positive correlations were found between fungal diversity and bacterial diversity, and between fungal richness and bacterial diversity, evenness, and richness (Spearman's correlation, permutation test, $p<0.05$, Supplementary Figure 11). In the post-treatment samples of FMT nonresponders and STD responders, the $\alpha$ diversity correlation between bacterial and fungal communities displayed a depletion of correlations between fungal richness and other bacterial and fungal $a$ diversity indexes. Correlations were completely abolished across the post-treatment samples of STD nonresponders.

Gut fungal dysbiosis correlates with FMT efficacy in a CDI mouse model. Our results, showing C. albicans as the most prominent species associated with treatment failure of FMT in CDI, suggested a possible causal relationship. This assumption is further supported by reports whereby CDI recurrence was observed after antibiotics treatment ${ }^{3,23}$, as antibiotics contribute to the expansion of Candida. To explore a potential causal relationship between C. albicans and an unfavorable FMT response, we assessed the efficacy of FMT in eliminating C. difficile using a C. difficile-induced diarrhea murine model in three groups of mice with a control group of CDI mice without FMT treatment (CDI): (i) mice infused with human stool preparation (CDI + FMT), (ii) mice colonized with C. albicans then infused with human stool preparation (CDI + CA + FMT), and (iii) mice infused with human stool preparation supplemented with $C$. albicans during fecal transplantation (CDI + FMT + CA) 
a
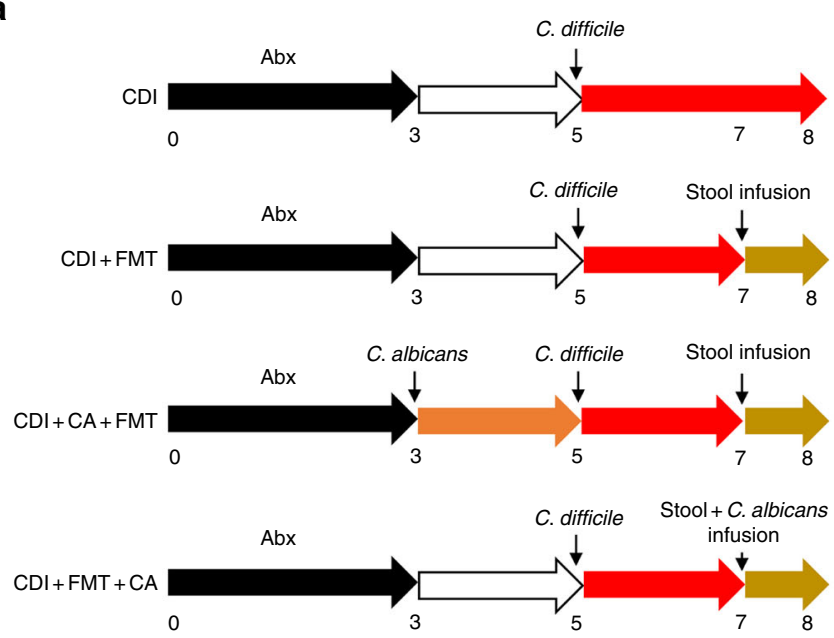

C
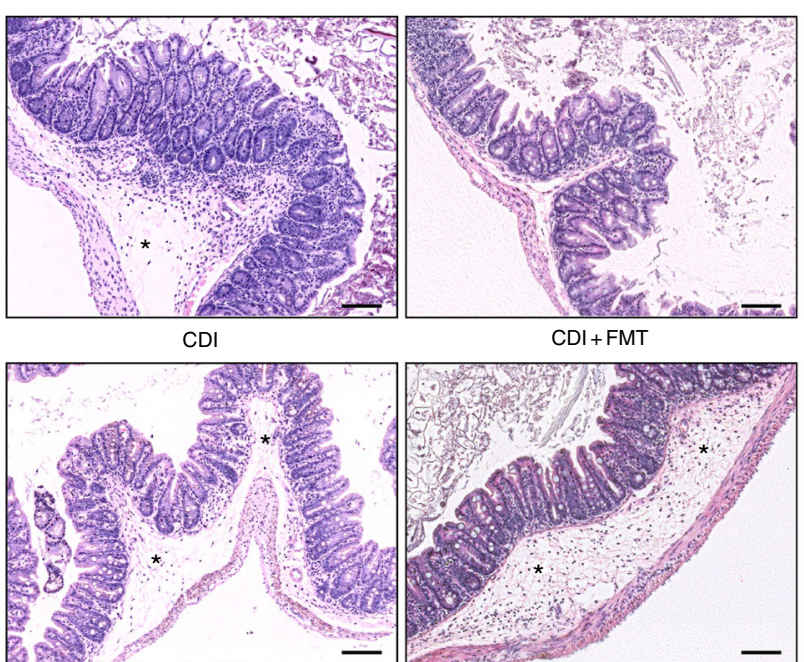

$\mathrm{CDI}+\mathrm{CA}+\mathrm{FMT}$

Constion b

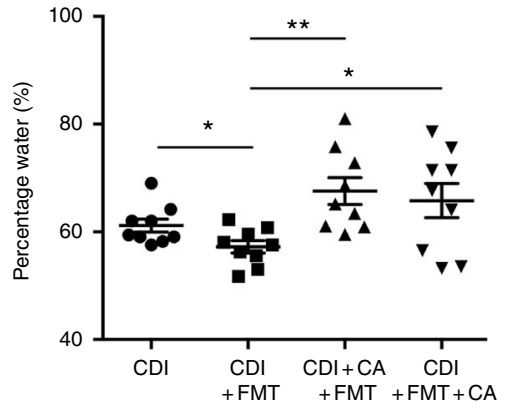

d

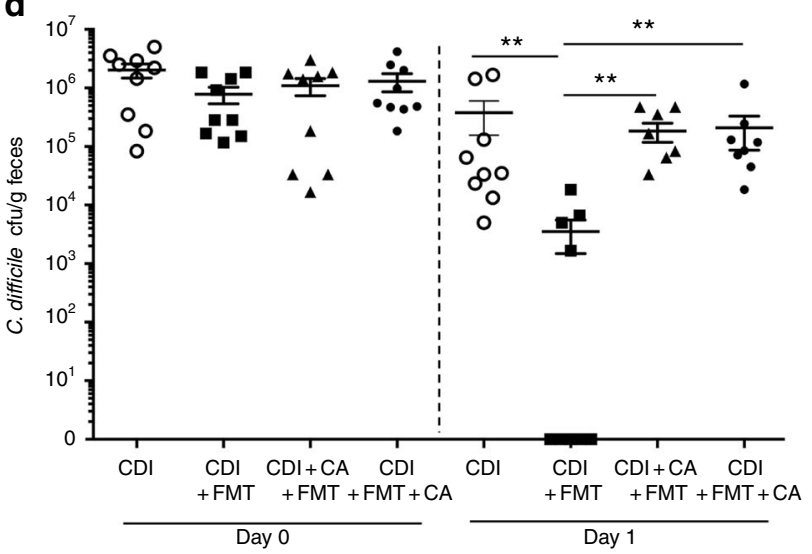

e

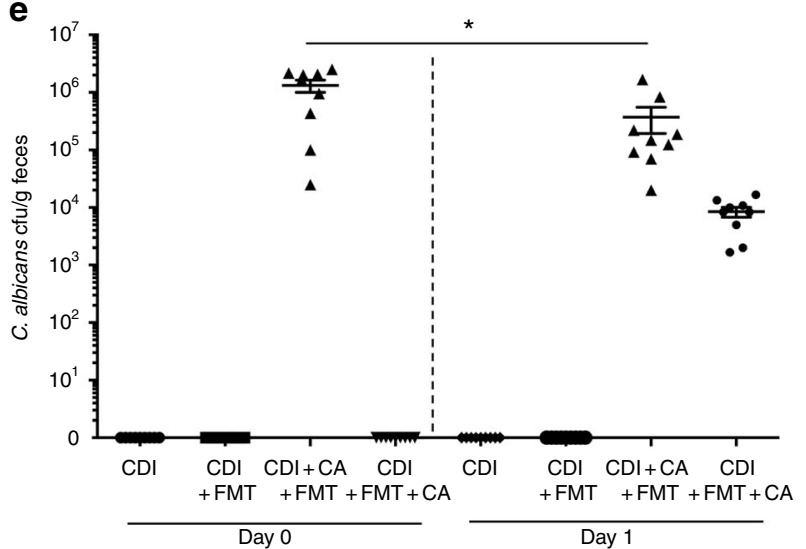

Fig. 5 C. albicans compromises FMT efficacy in eradicating C. difficile infection in mice. a Schematic diagram of C. albicans administration and stool infusion (FMT) in a murine $C$. difficile infection (CDI) model. Antibiotic treatment was ceased before gavage of C. albicans (CA) and C. difficile. Number denotes day across the timeline of the experiment. $\mathbf{b}$ Diarrhea in mice on day 1 after FMT. Stool percentage water was expressed as mean \pm s.e.m. Statistical significance represents comparisons by $t$ test, ${ }^{\star} p<0.05,{ }^{* \star} p<0.01$. c Representative H\&E-stained cecum sections on day 1 after stool infusion (asterisk denotes edema). Scale bar, $100 \mu \mathrm{m}$. Histological scores were assessed and expressed as mean \pm s.e.m. $n=6$ mice per group. Statistical significance represents comparisons by $t$ test, ${ }^{*} P<0.05,{ }^{\star \star} p<0.01,{ }^{* \star \star} p<0.001$. d Enumeration of $C$. difficile in mouse feces on day 0 before FMT and day 1 post-FMT ( $n=9$ mice per group). Statistical significance represents comparisons of FMT-treated mice with $C$. difficile infection versus other groups by unpaired Mann-Whitney test. ${ }^{\star} p<0.05,{ }^{\star \star} p<0.01$. e Enumeration of $C$. albicans in mouse feces both on day 0 before FMT and on day 1 post-FMT ( $n=9$ mice per group). Statistical significance represents comparison between $C$. albicans load on day 0 before FMT and day 1 post-FMT, by paired Mann-Whitney test. ${ }^{\star} p<0.05$. Dot graphs show means \pm s.e.m, performed at least two times independently 
(Fig. 5a). FMT was effective in ameliorating diarrhea, intestinal inflammation, and decreasing $C$. difficile burden, compared to CDI group, while no difference in $C$. difficile burden was observed among all groups before FMT (Fig. 5b-d). However, mice that was colonized with C. albicans prior to FMT or those infused with donor stool supplemented with $C$. albicans suffered significant diarrhea, intestinal inflammation, and augmented $C$. difficile burden post-FMT, when compared with mice administered with a single infusion of human stool (Fig. $5 b-d$ ). There were high levels of $C$. albicans in these recipient mice on day 1 post-FMT, though a decrease in C. albicans load was observed after FMT in mice colonized with $C$. albicans prior to FMT (Fig. 5e). We subsequently used an antifungal agent, fluconazole, to eradicate C. albicans in a group of recipient mice prior to human stool infusion (FMT) (Supplementary Figure 12a). We then compared C. difficile load after human stool infusion between mice with and without antifungal treatment. Antifungal treatment in recipient mice colonized with $C$. albicans before human stool infusion restored the efficacy of FMT in clearing $C$. difficile infection (Supplementary Figure 12b). These data support that C. albicans, either in recipient or in donor, can reduce the efficacy of FMT in clearing $C$. difficile, while antifungal treatment reestablishes its efficacy. Further research is needed to test whether this causal relationship, shown here in an animal model, applies also to patients with other diseases when doing FMT.

We also investigated the effects of Penicillium brocae and Aspergillus penicillioides, two filamentous fungi which were more enriched in healthy individuals than in CDI, on treating CDI during FMT (Supplementary Figure 13a). Mice that were colonized with $P$. brocae or A. penicillioides prior to FMT and mice that were supplemented with $A$. penicillioides during FMT showed higher levels of $C$. difficile burden compared with mice treated with FMT alone (Supplementary Figure 13b, on day 1 after FMT). However, these fungi-administered mice did not show any difference in symptoms of diarrhea on day 1 after FMT when compared with mice treated with FMT alone (Supplementary Figure 13c). These data suggest that different gut fungi may have disparate roles in FMT.

\section{Discussion}

This exploratory study, to our knowledge, is the first to characterize the gut mycobiota in CDI and to elucidate mycobiota alterations after FMT in relation to treatment outcome. Patients with CDI showed enteric fungal dysbiosis. Importantly, disease recurrence after FMT was associated with persistent fungal dysbiosis, low levels of donor-derived fungal colonization, high abundance of $C$. albicans in recipient stool, and presence of $C$. albicans in donor stool. Our observation that disease cure correlates with both fungal and bacterial colonization from the donor supports the idea that the gut mycobiota may play an important role in FMT outcome. In particular, we have shown that the abundance of fecal C. albicans, both in recipient before treatment and in donor, correlates with FMT outcome.

The role of fungal commensals in educating the human immune system has gained new appreciation in intestinal diseases. In the steady state, bacterial communities keep fungi in check in the gut. Fungi are major causes of infections among immunocompromised or hospitalized patients with serious underlying diseases and comorbidities ${ }^{24}$. Commensal bacteria inhibit C. albicans colonization through activation of HIF- $1 \alpha$ and LL- $37^{25}$. Antibiotic treatment selectively and effectively eradicates the bacterial community, but consequently leads to fungal outgrowth, particularly Candida species ${ }^{26,27}$. Antibiotics or immunosuppressants are effective in the short term but they likely compromise the immune system in the longer term. A compromised immune system creates a more favorable environment to expansion of Candida and overgrowth of Candida can alter the recovery of the gut bacteria microbiota after cessation of antibiotic treatment ${ }^{22,28}$. Host inflammation strongly promotes C. albicans colonization and conversely, C. albicans augments inflammation in a colitis murine mode ${ }^{29}$. C. albicans maintains commensalism or pathogenicity in host by modulating the local Th17/Treg balance ${ }^{30}$. In another aspect, it has been demonstrated that introduction of $C$. albicans into the bacterial microbiome of mice during a broad community disturbance has the potential to significantly alter the subsequent reassembly of the bacterial community as it recovers from that disturbance ${ }^{28}$. Taken together, our data from patients and from mouse studies support the hypothesis that an over-abundance of Candida species in recipients may contribute to FMT failure in CDI. Previous studies have shown that $C$. albicans and Candida are significantly enriched in a dextran sulfate sodium (DSS)-induced colitis mouse model as well as patients with IBD $^{31-33}$. Antifungal treatment decreased Candida prevalence and ameliorated inflammatory responses in DSS colitis mice ${ }^{33}$. However, disruption of fungal communities by long-term use of antifungals aggravated severity of DSS colitis and allergic airway ${ }^{34}$. Collectively, these data support the importance of the gut fungal-bacterial homeostasis in host health, which is also consistent with our data showing that FMT nonresponders display simultaneous alterations in fungal and bacterial $\alpha$-diversity when compared with responders.

In conclusion, gut mycobiota alterations in CDI patients correlate with FMT outcome, and our mouse assays support the idea that a causal relationship may exist. In particular, it is possible that $C$. albicans may contribute to CDI recurrence. Further research is needed to explore whether detailed characterization of both donor and recipient fecal mycobiomes may be useful for selection of "optimal" donors, and whether pre-FMT eradication of $C$. albicans in some recipients might increase FMT success rates in some cases.

\section{Methods}

Study subjects and treatment outcome. The current study was a substudy from a randomized controlled trial (RCT) of FMT versus vancomycin (STD) for patients with CDI. Consecutive CDI subjects enrolled into this RCT were invited to participate in a substudy of assessment of fecal microbiota. Patients were included if they had three or more loose or watery stools per day for at least two consecutive days or eight or more soft or loose stools in $48 \mathrm{~h}$ and a positive-stool test for $C$. difficile based on a two-step testing algorithm in our hospital, a positive-glutamate dehydrogenase screening test followed by a positive-polymerase chain reaction (PCR) test of C. difficile. A total of 31 subjects with CDI and 24 healthy household controls were recruited and stool samples at baseline were obtained for analyses of fungal and bacterial microbiomes. Among them, 24 CDI subjects consented to have stool samples collected serially after treatment for microbiome analysis. Totally, 16 CDI subjects were treated with FMT and 8 were treated with vancomycin, and they were followed up at baseline and at weeks 2, 4, 10, and 16 after treatment (Supplementary Figure 2). Subjects in FMT group received 5 days of vancomycin (125 $\mathrm{mg}$, four times daily) followed by donor infused stool via nasojejunal route and those who had STD received oral vancomycin $500 \mathrm{mg}$ orally four times per day for 10 days. A computer-generated randomization schedule was used to assign patients to the treatment sequences. All patients kept a stool diary and were questioned about stool frequency and consistency and medication use. The study was approved by The Joint Chinese University of Hong Kong, New Territories East Cluster Clinical Research Ethics Committee (The Joint CUHK-NTEC CREC, CREC Ref. No.: 2014.183-T; Clinical Trial registry, NCT02570477). We collected stool samples from new consecutive CDI patients. From this cohort, we included $12 \mathrm{CDI}$ patients with antibiotics exposure, $12 \mathrm{CDI}$ patients with no antibiotics exposure at inclusion, and an additional 17 healthy controls to determine effect of antibiotics on C. albicans. In addition, five new CDI subjects were treated with FMT (using two new donors with undetectable fecal C. albicans as determined by quantitative PCR) and consented to have stool samples collected serially after treatment for C. albicans quantitative analysis to confirm the findings.

Treatment response was defined as an absence of diarrhea or persistent diarrhea that could be explained by other causes with a negative stool test for $C$. difficile toxin, while relapse was defined as diarrhea with a positive-stool test for $C$. difficile toxin. Treatment cure is defined as symptom resolution and a negative $C$. difficile toxin in stool until the last follow-up (last follow-up is referred to as the last stool 
collection time-point, as shown in Supplementary Figure 2). Out of 16, 9 subjects who had FMT (FMT1-FMT9), and 5 of the 8 patients (STD1-STD5), who had vancomycin were cured of CDI (termed responders, Supplementary Data 3) at a median follow-up of 16 weeks. CDI recipients FMT11 and FMT12 shared the same donor, and this donor was termed "Donor11". Clinical data of the subjects and collected stool samples are shown in Supplementary Datas 3 and 4. None of the patients had received antibiotics or proton pump inhibitors after FMT.

\section{Study design}

Patient inclusion criteria.

1. C. difficile infection was defined as diarrhea ( $\geq 3$ soft, loose or watery stools per day for at least 2 consecutive days or $\geq 8$ soft or loose stools in $48 \mathrm{~h}$ ).

2. A positive-stool test for C. difficile toxin (on a two-step testing algorithm, a positive-GDH screening test followed by a positive-PCR test of C. difficile).

3. Age $\geq 18$

4. Written informed consent obtained.

\section{Patient exclusion criteria.}

1. The presence of human immunodeficiency virus (HIV) infection with a CD4 count of less than 240 .

2. Pregnancy.

3. Gastrointestinal bleeding.

4. Acute coronary syndrome.

Donor screening. Donors included individuals who are spouses or partners, firstdegree relatives, other relatives, friends, and individuals unknown to the patient. They were screened with a questionnaire and excluded if they had taken antibiotics within the preceding 3 months; were on major immunosuppressive agents, including chemotherapeutic agents; had known or recent exposure to HIV, hepatitis B or C; had a current communicable disease; participated in high-risk sexual behaviors; used illicit drugs; traveled within 6 months to areas with endemic diarrheal illnesses; or had history of IBD, irritable bowel syndrome or chronic diarrhea, gastrointestinal malignancy or polyposis. In addition, donor was screened for HBsurface Ag, Anti-HBc, Anti-HCV, Anti-HIV, Syphilis EIA, stool microscopy, culture and sensitivity, stool cyst, ova, parasite, norovirus, and C. difficile (cytotoxin and PCR assay). All subjects and collected stool samples are listed in Supplementary Data 4.

The donors for the FMT group were healthy household controls and the donor stool samples analyzed were the same samples used for FMT. All subjects provided written informed consent.

Family members provided donor stool for subjects randomized to FMT arm. Cure after FMT or vancomycin therapy was defined as symptom resolution and negative C. difficile toxin in stool at last follow-up by PCR assay. Relapse was defined as diarrhea with a positive-stool test for $C$. difficile toxin.

This was a randomized, but not blinded study. However, for mycobiome and bacterial microbiome analyses on stool samples, assessments were initially performed by analysts who were blinded to the clinical outcome of the studied subjects. When the profiled mycobiome and bacterial microbiome data were available for each individual subject, correlation was then made to associate microbiome profiles with treatment outcomes of subjects.

Infusion of donor stool. In subjects who received FMT, a nasoduodenal tube was inserted with radiology guidance. Donor feces was diluted with $500 \mathrm{ml}$ of sterile saline $(0.9 \%)$, blended and the supernatant was strained with filter paper and poured in a sterile bottle. Within $6 \mathrm{~h}$ after collection of feces by the donor, the solution was infused through a nasoduodenal tube $(2-3 \mathrm{~min}$ per $50 \mathrm{ml})$. The tube was removed $30 \mathrm{~min}$ after the infusion, and patients were monitored for $2 \mathrm{~h}$. In subjects who received FMT, a minimum of $50 \mathrm{~g}$ of donor stool was collected on the same day of infusion and used within $6 \mathrm{~h}$ of collection.

Fecal DNA extraction. Fecal DNA was isolated as described below. A $100 \mathrm{mg}$ fecal

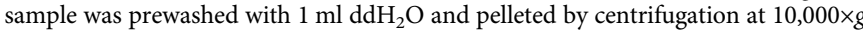
for $1 \mathrm{~min}$. The fecal pellet was resuspended in $800 \mu \mathrm{l} \mathrm{TE}$ buffer ( $\mathrm{pH} 7.5)$, supplemented with $1.6 \mu \mathrm{l} 2$-mercaptoethanol and $500 \mathrm{U}$ lyticase (Sigma), and incubated at $37^{\circ} \mathrm{C}$ for $60 \mathrm{~min}$. The sample was then centrifuged at $10,000 \times \mathrm{g}$ for $2 \mathrm{~min}$ and fecal DNA was subsequently extracted from the pellet using ZR Fecal DNA miniPrep kit (Zymo Research, Orange, CA) according to the protocal. Briefly, fecal pellet was added to the BashingBeadLysis Tube with $750 \mu \mathrm{L}$ Lysis solution, and then processed at maximum speed for $10 \mathrm{~min}$. The lysates were centrifuged at $\geq 10,000 \times \mathrm{g}$ for 1 min. The supernatant was transferred to a Zymo-Spin ${ }^{\mathrm{TM}}$ IV Spin Filter in a collection tube and centrifuged at $7000 \times g$ for $1 \mathrm{~min}$. About $1200 \mu \mathrm{l}$ of fecal DNA binding buffer was added to the filtrate in the collection tube, followed by concentration and purification in a new filter tube. Finally, a total of $50 \mu$ l eluted DNA with a concentration at $20-100 \mathrm{ng} / \mu \mathrm{l}$ was prepared for each sample.
Fungal ITS2 sequencing and quality control. The final fecal DNA for fungal sequencing was amplified based upon internal transcribed spacer 2 (ITS2) region using primers as below and PrimeSTAR HS DNA Polymerase kit (TaKaRa, Japan) The primer pairs are ITS2-F: 5'-GCATCGATGAAGAACGCAGC-3', ITS2-R: 5'TCCTCCGCTTATTGATATGC- $3^{\prime}$. ITS2 amplicons were generated with 38 cycles of 3-step PCR: $98^{\circ} \mathrm{C} 10 \mathrm{~s}, 59^{\circ} \mathrm{C} 10 \mathrm{~s}$, and $72^{\circ} \mathrm{C} 30 \mathrm{~s}$. PCR samples were then sequenced on the Illumina MiSeq PE300 platform $(2 \times 300 \mathrm{bp}$, BGI, China), $151,524 \pm 97,694$ (mean \pm SD) clean sequences obtained on average (sequence statistics in Supplementary Data 5).

Raw reads were filtered by SOAPnuke (v 1.5.3) (http://soap.genomics.org.cn/) developed by BGI as follows: (i) adapters removed, (ii) read removed if $\mathrm{N}$ base is more than $3 \%$ of the read, (iii) read removed if bases with quality low than 20 were more than $40 \%$ of read, and (iv) all duplicates removed. Quality control and data analysis were further implemented in PIPITS (v 1.4.5) $)^{35}$. Briefly, PIPITS_PREP prepares raw reads from Illumina MiSeq sequencers for ITS extraction; PIPITS_FUNITS extracts ITS2 from the reads; and PIPITS_PROCESS analyses the reads to produce OTU abundance tables and the Ribosomal Database Project (RDP) taxonomic assignment table for downstream analysis. The quality trimmed and ITS2 extracted reads were aligned to fungi UNITE database exploiting RDP classifier 2.10 for taxonomic assignment to produce OTU abundance table (based on sequence identity $\geq 97 \%$ identity) and phylotype abundance tables at different taxonomic levels, for downstream analysis.

The fungal OTU and phylotype abundance data were imported into $R$ 3.2.3. Richness, diversity, and evenness calculation were performed using the estimated richness function of the phyloseq package. Spearman correlation and their significance were calculated using the cor and cor.test functions in R, respectively. For the fungal-bacterial taxa comparisons, Spearman correlations were calculated for the relative abundance of the differentially presented fungal taxa and the bacterial taxa determined to be significantly associated with disease by Lefse analysis. Correlation plots were generated using the corrplot package in R. Heatmaps were generated using the pheatmap package in R.

\section{Quantitative PCR for detection of total fungal load in human fecal DNA} samples. Total fungal loads in human stools were quantified by TaqMan qPCR analysis (Premix Ex Taq ${ }^{\mathrm{TM}}$, TaKaRa) of extracted human fecal DNA using primers $^{36}$ : Fungi-quant-F 5'-GGRAAACTCACCAGGTCCAG-3'; Fungi-quant-R 5' GSWCTATCCCCAKCACGA-3', and probe: 5'-TGGTGCATGGCCGTT-3'.

Quantitative PCR for detection of C. albicans in human fecal DNA samples. $C$ albicans loads in human stools were quantified by qPCR analysis (SsoAdvanced SYBR Green Supermix, Bio-Rad) of extracted human fecal DNA using C. albicans specific primers: C.albicans-F 5'-CCTGTTTGAGCGTCGTTTCTC-3'; C. albicansR 5'-TTTGGTTAGACCTAAGCCATTGTCA-3'. C. albicans absolute abundance was determined using standard curve constructed with reference genomic DNA (gDNA) of C. albicans.

LefSe linear discriminant analysis and multivariate analysis. To compare differences in the configurations of fungal and bacterial microbiomes between CDI patients and healthy controls, between FMT responders and nonresponders, between FMT treatment and vancomycin (STD) treatment, LefSe analyses were performed on the Huttenhower lab Galaxy server (http://huttenhower.sph.harvard. edu/galaxy/) by importing the fungal and bacterial relative abundance values and associated sample metadata, with FDR-adjusted $q$ value $<0.05$ considered significant and effect size calculated. MaAsLin (Multivariate Analysis by Linear Models) was implemented to identify associations between clinical metadata (age, gender, household, CDI severity, and underlying IBD) and fungal community abundance matrix on the Huttenhower lab Galaxy server (http://huttenhower.sph. harvard.edu/galaxy/).

Nonmetric multidimensional scaling analysis. The difference in fecal fungal community structures between controls and CDI was performed via NMDS plot based upon Bray-Curtis dissimilarities among all subjects. The two ellipses correspond to the fecal mycobiota community dispersion range within each group, controls and CDI, respectively, drawn using the function veganCovEllipse in the Package Vegan in $R$ basing upon the Least-Squares criterion for estimation of the best fit to an ellipse from a given set of mycobiota communities.

Bacterial 16S rRNA sequencing and data analysis. The final fecal DNA samples were subject to bacterial 16S rRNA V4 region amplification and sequenced on the Illumina MiSeq PE250 platform ( $2 \times 250$ bp, BGI, China), 132,081 $\pm 65,429$ (number \pm SD) sequences obtained on average (sequence statistics in Supplementary Data 6). Quality control and data analysis were implemented in mothur (v $1.38 .0)$ as previously described ${ }^{37}$. Any sequences with ambiguous bases and anything longer than $275 \mathrm{bp}$ were removed, and aligned against the nonredundant Greengenes database (v 13.8) ${ }^{38}$ using the NAST algorithm. Any sequences that failed to align with the V3-4 region were discarded. The remaining sequences were trimmed to the same alignment coordinates over which they fully overlapped, followed by removal of homopolymers and detection for the presence of chimeras by UChime. 
The resulting sequences were classified against the Greengenes database and annotated with deepest level taxa represented by pseudo-bootstrap confidence scores of at least $80 \%$ averaged over 1000 iterations of the naive Bayesian classifier. Any sequences that were classified as either being originated from archaea, eukarya, chloroplasts, mitochondria, or unknown kingdoms, were removed. The annotated sequences were assigned to phylotypes according to their consensus taxonomy with which at least $80 \%$ of the sequences agreed. Closed reference OTUs sharing $97 \%$ identity were clustered as well and assigned taxonomy according to the Greengenes database. LefSe analysis was performed to define bacterial taxa associated with CDI and healthy controls. The relative abundance of these abundance-differential taxa identified by LefSe in pre-FMT baseline samples and post-FMT last follow-up samples were plotted using pheatmap $\mathrm{R}$ package.

Calculation of donor transferred OTUs in recipients. In samples after FMT, if a fungal or bacterial OTU was not present in the recipient baseline sample but present both in the corresponding donor baseline sample and in the recipient postFMT sample, the OTU was defined as "donor derived"; if an OTU was not present in the corresponding donor baseline sample, but detected both in the recipient baseline sample and in the recipient post-FMT sample, the OTU was defined as "recipient exclusive"; if an OTU was present across the recipient baseline sample, the recipient post-FMT sample and the corresponding donor baseline sample, the OTU was defined as "donor-recipient co-existed".

Mouse husbandry and model of $\boldsymbol{C}$. difficile infection. Studies were conducted on 4- to 6-week-old female C57BL/6 that were reared in groups of nine. Individual mice were randomized after arrival. Mice were subjected to a previously described model of $\mathrm{CDI}^{39}$. Briefly, mice were given an antibiotic cocktail of kanamycin $(0.4$ $\mathrm{mg} / \mathrm{mL})$, gentamicin $(0.035 \mathrm{mg} / \mathrm{mL})$, colistin $(850 \mathrm{U} / \mathrm{mL})$, metronidazole $(0.215$ $\mathrm{mg} / \mathrm{mL}$ ), and vancomycin $(0.045 \mathrm{mg} / \mathrm{mL}$ ) (all antibiotics were purchased from Sigma-Aldrich, St. Louis, MO) in their drinking water for 3 days. Mice were then given 2 days of recovery before administration of $10^{7}$ spores of $C$. difficile (strain HK31423, Ribotype 002, a clinical isolate during an outbreak at Prince of Wales Hospital in Hong Kong) in phosphate-buffered saline (PBS) via oral gavage. Animal grouping and research scheme were designed as shown in Fig. 4a. On day 1 poststool infusion, diarrhea was evaluated by stool water content, calculated as stool weight loss after air drying at $70^{\circ} \mathrm{C}$ for $4 \mathrm{~h}$. Cecums were harvested, fixed in $4 \%$ formalin solution and embedded in paraffin on day 1 after FMT. Sections were stained with hemotoxylin and eosin for histological assessment ${ }^{40}$. In experiment for investigating the effect of vancomycin administration on the presence of Candida in mice, seven 4- to 6-week old female C57BL/6 mice were given vancomycin $(0.1 \mathrm{mg} / \mathrm{mL})$ in their drinking water for 3 days. Stool samples before and after vancomycin regime were collected and subsequently determined for Candida levels by qPCR analysis (SsoAdvanced SYBR Green Supermix, Bio-Rad) using primers ${ }^{41}$ : pan-Candida-F 5'-GCAAGTCATCAGCTTGCGTT-3'; pan-Candida-R $5^{\prime}$-TGCGTTCTTCATCGATGCGA- ${ }^{\prime}$. All animal experiments were approved and performed in compliance with the Animal Experimentation Ethics Committee (AEEC) of The Chinese University of Hong Kong.

C. albicans administration and donor stool infusion in mice. C. albicans (10231, from ATCC, USA) was administered to mice $\left(2 \times 10^{8} \mathrm{cfu}\right.$ per mouse) via gavage after 3-day antibiotic treatment or supplemented in donor stool slurry at the time of donor stool infusion. Human stool from a healthy volunteer (Chinese, male, age 28 years), without presence of $C$. albicans as measured by qPCR, was obtained with informed consent. For stool microbiota infusions, approximately $2 \mathrm{~g}$ of stool samples were cut in an anaerobic chamber and suspended in $10 \mathrm{ml}$ of PBS. Mice were colonized by oral gavage of $150 \mu \mathrm{l}$ of fecal slurry with or without supplementation of $C$. albicans on day 2 after $C$. difficile challenge. For antifungal experiment, animal grouping and research scheme were designed as shown in Supplementary Figure 12a. Mice was initially colonized with C. albicans $\left(2 \times 10^{8}\right.$ cfu per mouse) after 3 days of antibiotic cocktail treatment in the drinking water, followed by 4 days of fluconazole treatment supplemented in the drinking water $(0.5 \mathrm{mg} / \mathrm{mL}$, Sigma). Then the mice were subjected to $C$. difficile administration $\left(10^{7}\right.$ spores per mice) through gavage after a consecutive 1.5-day antibiotic cocktail- and 1.5-day free water-drinking. Human stool infusion was performed 2 days later after C. difficile gavage. Both C. difficile load and C. albicans load were enumerated by cultivation on day 0 before FMT and day 1 after FMT.

Quantification of $\mathbf{C}$. difficile and C. albicans burdens in mouse feces. Mouse stool were collected both before and after stool infusion. Fecal C. difficile and $C$. albicans burdens on day 0 before and day 1 after stool infusion were measured by cultivation. Samples were diluted in PBS and, respectively, plated on taurocholate cycloserine cefoxitin fructose agar for quantification of $C$. difficile burden, on Sabouraud dextrose agar (SDA) for quantification of C. albicans load. Stool samples prior to C. albicans colonization from antibiotic-treated mice were plated on SDA to ensure that mice were $C$. albicans culture negative.

Administration of $\boldsymbol{P}$. brocae, and $\boldsymbol{A}$. penicillioides, and donor stool infusion in mice. A 4- to 6-week-old female C57BL/6 that were reared in groups of eight. Animal grouping and research scheme were designed as shown in Supplementary
Figure 13a. P. brocae (CBS116042, from Westerdijk Fungal Biodiversity Institute, Netherlands) and A. penicillioides (CBS112373, from Westerdijk Fungal Biodiversity Institute, Netherlands) were administered to mice, respectively (approximately $0.5 \mathrm{~g} / \mathrm{kg}$ ), via gavage after 3-day antibiotic treatment or supplemented in donor stool slurry at the time of donor stool infusion. Colonization of P. brocae or A. penicillioides in mice was confirmed by PCR with detectable genomic DNA of respective species in fecal DNA after oral administration of each fungus. For stool microbiota infusions, approximately $2 \mathrm{~g}$ of stool samples were cut in an anaerobic chamber and suspended in $10 \mathrm{ml}$ of PBS. Mice were colonized by oral gavage of $150 \mu \mathrm{l}$ of fecal slurry with or without supplementation of these fungi on day 2 after C. difficile challenge.

\section{Data availability}

Sequence data and accompanying metadata have been deposited to the NCBI Sequence Read Archive under BioProject accession numbers PRJNA419097 and PRJNA419104.

Received: 13 July 2018 Accepted: 15 August 2018

Published online: 10 September 2018

\section{References}

1. Smits, L. P., Bouter, K. E. C., de Vos, W. M., Borody, T. J. \& Nieuwdorp, M. Therapeutic potential of fecal microbiota transplantation. Gastroenterology 145, 946-953 (2013).

2. Vrieze, A. et al. Transfer of intestinal microbiota from lean donors increases insulin sensitivity in individuals with metabolic syndrome. Gastroenterology 143, 913-916 (2012).

3. van Nood, E. et al. Duodenal infusion of donor feces for recurrent Clostridium difficile. New Engl. J. Med. 368, 407-415 (2013).

4. Lee, C. H. et al. Frozen vs. fresh fecal microbiota transplantation and clinical resolution of diarrhea in patients with recurrent Clostridium difficile infection: a Randomized Clinical Trial. J. Am. Med. Assoc. 315, 142-149 (2016).

5. Drekonja, D. et al. Fecal microbiota transplantation for Clostridium difficile infection: a systematic review. Ann. Intern. Med. 162, 630-U230 (2015).

6. Paramsothy, S. et al. Faecal microbiota transplantation for inflammatory bowel disease: a systematic review and meta-analysis. J. Crohns Colitis 11, 1180-1199 (2017).

7. Costello, S. P. et al. Systematic review with meta-analysis: faecal microbiota transplantation for the induction of remission for active ulcerative colitis. Aliment. Pharmacol. Ther. 46, 213-224 (2017).

8. Kelly, C. R., Kahn, S. A. \& Kashyap, P. Update on fecal microbiota transplantation 2015: indications, methodologies, mechanisms, and outlook (vol 149, p. 223, 2015). Gastroenterology 149, 1644-1644 (2015).

9. Khoruts, A. \& Sadowsky, M. J. Therapeutic transplantation of the distal gut microbiota. Mucosal Immunol. 4, 4-7 (2011).

10. Manichanh, C. et al. Reshaping the gut microbiome with bacterial transplantation and antibiotic intake. Genome Res. 20, 1411-1419 (2010).

11. Li, S. S. et al. Durable coexistence of donor and recipient strains after fecal microbiota transplantation. Science 352, 586-589 (2016).

12. Khoruts, A. \& Sadowsky, M. J. Understanding the mechanisms of faecal microbiota transplantation. Nat. Rev. Gastroenterol. Hepatol. 13, 508-516 (2016).

13. Zuo, T. et al. Bacteriophage transfer during faecal microbiota transplantation in Clostridium difficile infection is associated with treatment outcome. Gut 67, 634-643 (2017)

14. Chehoud, C. et al. Transfer of viral communities between human individuals during fecal microbiota transplantation. mBio 7, e00322-16 (2016).

15. Broecker, F. et al. Long-term changes of bacterial and viral compositions in the intestine of a recovered Clostridium difficile patient after fecal microbiota transplantation. Mol. Case Stud. 2, a000448 (2016).

16. Limon, J. J., Skalski, J. H. \& Underhill, D. M. Commensal fungi in health and disease. Cell Host Microbe 22, 156-165 (2017)

17. Iliev, I. D. \& Leonardi, I. Fungal dysbiosis: immunity and interactions at mucosal barriers. Nat. Rev. Immunol. 17, 635-646 (2017).

18. Jiang, T. T. et al. Commensal fungi recapitulate the protective benefits of intestinal bacteria. Cell Host Microbe 22, 809-816 e804 (2017).

19. Leffler, D. A. \& Lamont, J. T. Clostridium difficile infection. New Engl. J. Med 372, 1539-1548 (2015).

20. Stevens, V., Dumyati, G., Fine, L. S., Fisher, S. G. \& Van Wijngaarden, E. Cumulative antibiotic exposures over time and the risk of Clostridium difficile infection. Clin. Infect. Dis. 53, 42-48 (2011).

21. Kobayashi-Sakamoto, M., Tamai, R., Isogai, E. \& Kiyoura, Y. Gastrointestinal colonisation and systemic spread of Candida albicans in mice treated with antibiotics and prednisolone. Microb. Pathog. 117, 191-199 (2018).

22. Mason, K. L. et al. Candida albicans and bacterial microbiota interactions in the cecum during recolonization following broad-spectrum antibiotic therapy. Infect. Immun. 80, 3371-3380 (2012). 
23. Leffler, D. A. \& Lamont, J. T. Clostridium difficile infection. New Engl. J. Med. 373, 287-288 (2015).

24. Flevari, A., Theodorakopoulou, M., Velegraki, A., Armaganidis, A. \& Dimopoulos, G. Treatment of invasive candidiasis in the elderly: a review. Clin. Interv. Aging 8, 1199-1208 (2013).

25. Fan, D. et al. Activation of HIF-1 alpha and LL-37 by commensal bacteria inhibits Candida albicans colonization. Nat. Med. 21, 808-814 (2015).

26. Mason, K. L. et al. Interplay between the gastric bacterial microbiota and Candida albicans during postantibiotic recolonization and gastritis. Infect. Immun. 80, 150-158 (2012).

27. Dollive, S. et al. Fungi of the murine gut: episodic variation and proliferation during antibiotic treatment. PLoS One 8, e71806 (2013).

28. Downward, J. R. E., Falkowski, N. R., Mason, K. L., Muraglia, R. \& Huffnagle, G. B. Modulation of postantibiotic bacterial community reassembly and host response by Candida albicans. Sci. Rep. 3, 2192 (2013).

29. Jawhara, S. et al. Colonization of mice by Candida albicans is promoted by chemically induced colitis and augments inflammatory responses through galectin-3. J. Infect. Dis. 197, 972-980 (2008).

30. Bonifazi, P. et al. Balancing inflammation and tolerance in vivo through dendritic cells by the commensal Candida albicans. Mucosal Immunol. 2, 362-374 (2009).

31. Sokol, H. et al. Fungal microbiota dysbiosis in IBD. Gut 66, 1039-1048 (2017).

32. Sartor, R. B. \& Wu, G. D. Roles for intestinal bacteria, viruses, and fungi in pathogenesis of inflammatory bowel diseases and therapeutic approaches. Gastroenterology 152, 327-339 (2017).

33. Iliev, I. D. et al. Interactions between commensal fungi and the C-type lectin receptor dectin-1 influence colitis. Science 336, 1314-1317 (2012).

34. Wheeler, M. L. et al. Immunological consequences of intestinal fungal dysbiosis. Cell Host Microbe 19, 865-873 (2016).

35. Gweon, H. S. et al. PIPITS: an automated pipeline for analyses of fungal internal transcribed spacer sequences from the Illumina sequencing platform. Methods Ecol. Evol. 6, 973-980 (2015).

36. Liu, C. M. et al. FungiQuant: a broad-coverage fungal quantitative real-time PCR assay. BMC Microbiol. 12, 255 (2012).

37. Schloss, P. D. et al. Introducing mothur: open-source, platform-independent, community-supported software for describing and comparing microbial communities. Appl. Environ. Microb. 75, 7537-7541 (2009).

38. McDonald, D. et al. An improved Greengenes taxonomy with explicit ranks for ecological and evolutionary analyses of bacteria and archaea. ISME J. 6, 610-618 (2012).

39. Chen, X. H. et al. A mouse model of Clostridium difficile-associated disease. Gastroenterology 135, 1984-1992 (2008).

40. Erben, U. et al. A guide to histomorphological evaluation of intestinal inflammation in mouse models. Int. J. Clin. Exp. Pathol. 7, 4557 (2014).
41. Zhang, J. et al. Development of Candida-specific real-time PCR assays for the detection and identification of eight medically important Candida species. Microbiol. Insights 9, S38517 (2016). MBI.

\section{Acknowledgments}

We acknowledge the funding sources from Hong Kong Society of Gastroenterology, and a seed fund for Gut Microbiome Research provided by the Faculty of Medicine, The Chinese University of Hong Kong.

\section{Author contributions}

T.Z. performed the experiments, data analysis and drafted the manuscript. S.H.W. performed FMT in clinic, helped data analysis, and revised the manuscript. K.C. and W.T. collected the clinical samples and data. C.P.C. and F.Z. helped with DNA sample preparation and data validation. J.Y.L.C. and W.T. managed the clinical practice and clinical database. P.K.S.C., J.C.Y.W., K.L., and R.L. assisted in FMT and revised the manuscript. F.K.L.C., J.Y., and J.J.Y.S. provided critical comments on the manuscript. S.C.N. designed, supervised the study and wrote the manuscript.

\section{Additional information}

Supplementary Information accompanies this paper at https://doi.org/10.1038/s41467018-06103-6.

Competing interests: The authors declare no competing interests.

Reprints and permission information is available online at http://npg.nature.com/ reprintsandpermissions/

Publisher's note: Springer Nature remains neutral with regard to jurisdictional claims in published maps and institutional affiliations.

Open Access This article is licensed under a Creative Commons Attribution 4.0 International License, which permits use, sharing, adaptation, distribution and reproduction in any medium or format, as long as you give appropriate credit to the original author(s) and the source, provide a link to the Creative Commons license, and indicate if changes were made. The images or other third party material in this article are included in the article's Creative Commons license, unless indicated otherwise in a credit line to the material. If material is not included in the article's Creative Commons license and your intended use is not permitted by statutory regulation or exceeds the permitted use, you will need to obtain permission directly from the copyright holder. To view a copy of this license, visit http://creativecommons.org/ licenses/by/4.0/.

(c) The Author(s) 2018 